that coronal holes have an almost constant rotation rate which, with the sector structure of interplanetary fields, suggests the presence of cells elongated parallel to the rotation axis of the Sun. There is as yet no adequate theory of vigorous convection in a compressible fluid, though the interaction between convection and rotation has been studied for incompressible fluids. B. R. Durney (National Center for Atmospheric Research, Boulder, Colorado) found that the observed uniformity of the energy flux at the solar surface seemed incompatible with an angular velocity that increases inwards (as required for dynamo models). P. A. Gilman (NCAR) described numerical experiments on convection in a rotating spherical shell which led to large variations of angular velocity, though not necessarily to an equatorial acceleration. It is through such large scale computations that further theoretical progress must be made.

Turbulent diffusion is an essential feature of any solar dynamo, though there is no complete theory for the reconnection of magnetic fields on a sufficiently rapid time scale. The generally accepted view is that turbulence can bring opposing fields close enough together for dynamically driven reconnection to take place. E. R. Priest (St. Andrew's University) and A. M. Soward (Newcastle University) demonstrated that rapid reconnection can occur at rates up to the Alfvèn speed. J. H. Piddington (CSIRO, Sydney) dissented from the orthodox approach. He believes that turbulence may redistribute magnetic flux but is unable to destroy it. Indeed. his magnetic fields form isolated flux ropes that control the pattern of cellular convection (instead of being concentrated by the motion). So he has revived an alternative model of the solar cycle, relying on a primordial steady poloidal field from which the alternating toroidal field is generated by some form of torsional oscillation.

\section{Immunological tolerance}

\section{from a Correspondent}

A symposium on Immunological Tolerance was held at Reinhardsbrunn, GDR on October 14-17. It was sponsored by the International Union of Immunological Societies and the World Health Organization.

THE mechanisms of antigen-induced unresponsiveness (immunological tolerance) are still controversial: tolerance has been ascribed either to "deletion" of relevant lymphocyte clones, or to active suppression of these clones by other lymphocytes ( $\mathrm{T}$ suppressor cells) or by humoral "blocking factors".

The classical concept of clonal deletion in tolerance to transplantation antigens, first propounded by Sir MacFarlane Burnet and others, has withstood the test of time. Experiments by $\mathrm{H}$. von Boehmer and J. Sprent (Basel Institute for Immunology) with mouse radiation chimaeras $\left(\mathrm{F}_{1}\right.$ hybrid mice reconstituted with bone marrow cells from both parents), provide clear evidence that self-tolerance in these animals is due to clonal elimination and not to suppression.

Evidence for blocking factors in the maintenance of tolerance was also presented, however. H. Wekerle (Max Planck Institut, Freiburg) has induced autoreactive lymphocytes by coculturing lymph node cells and testicular cells from the same rat. The cytostatic activity of these cells is blocked by an unknown factor present ia autologous serum, and to a lesser degree by serum from allogeneic rats. M. Embleton (Cancer Research Campaign Laboratories, Nottingham) discussed experiments showing that rats developing hepatomas first have free tumour antigen in their serum, and then antigen-antibody complexes. The latter are effective in "blocking" the activity of effector lymphocytes, and disappear from the circulation within 3 days of the excision of the tumour.

Inhibition of lymphocyte effector function by $\mathrm{T}$ suppressor lymphocytes was a major topic of discussion. Both K. Eichmann (University of Cologne) and Leonore Herzenberg (Stanford University) presented evidence that suppressor $\mathrm{T}$ cells act by inhibiting the function of helper $T$ cells (cells which help B lymphocytes to make antibody). Data from A. Basten (University of Sydney) suggest, however, that under some conditions the target of suppression may be the antibody-forming cell precursor (B cell).

There were, nevertheless, close parallels between these three systems. Thus, suppressor cells seem to belong to a discrete $T$ cell subpopulation-bearing Ia antigens, distinctive Ly antigens, and with a characteristic tissue distribution. Most strikingly, both Eichmann's and Herzenberg's work suggests that there are categories of helper $T$ cells which only interact with a restricted $\mathrm{B}$ cell subset-those destined to produce a single allelic variant of one immunoglobulin subclass (Herzenberg) or a single idiotype (Eichmann). These specialised helper cells seemed to be the targets of suppression.

The general role of suppressor cells in tolerance remains unresolved. Basten presented clear evidence that although mice made tolerant with human gamma globulin developed suppressor cells, the animals remained tolerant long after the suppressors had disappeared. For the present one may conclude that, although suppressor cells are demonstrable in a number of tolerance models, they have not been shown to be obligatory either in the induction or maintenance of unresponsiveness. Rather, they may fulfil a more subtle homoeostatic function.

One session was devoted to tolerance induced by "suiciding" specific immune responses by attaching a lethal agent to an antigen, so that the complex inactivates clones of cells bearing receptors for that antigen. This conceptually attractive approach, with obvious potential therapeutic applications, has hitherto been largely restricted to animal models using highly radiolabelled antigens. Recently, however, $H$. Ambrosius and his collaborators at the University of Leipzig have been testing various immunosuppressive agents, in particular 6-mercaptopurine, coupled to protein antigens. Such conjugates specifically suppress anti-protein antibody synthesis in guinea pigs. D. A. L. Davies (Searle Research Laboratories, High Wycombe) is studying the effects of a phenylalanine mustard coupled to antitumour antibody on the growth of a transplantable mouse lymphoma. The results are encouraging: administration of the drug or antibody alone is ineffective, giving the drug at the same time as antibody is effective, while the covalent drug-antibody conjugate is even more effective.

The success of this meeting was undoubtedly partly due to the fact that it brought together experts in various aspects of tolerance, and enabled them to find common ground for a variety of phenomena. Most importantly, however, it also provided a unique noportunity for immunologists from the East and the West to meet and exchange ideas.

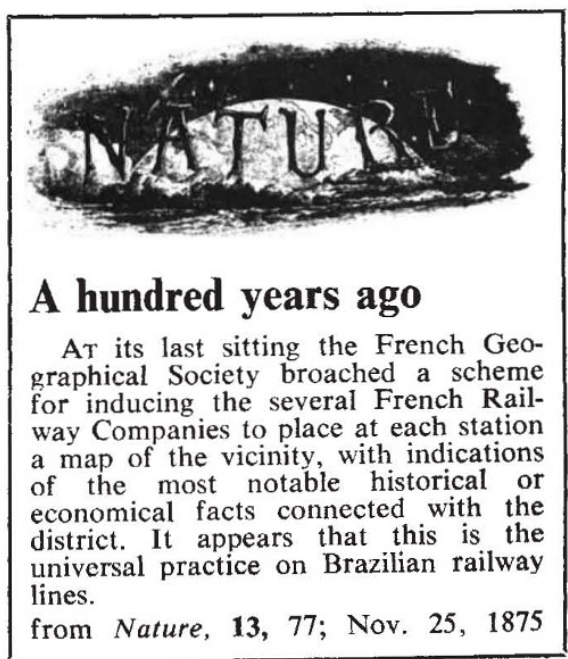

